\title{
Estudo experimental do efeito da argilosidade sobre As Propriedades Físicas De Solos Através Da Técnica De Ressonância Magnética Nuclear.
}

Calvin Tamanqueira do Couto, Gleide Alencar do Nascimento Dias, Livia Borges Pessanha, Universidade Federal do Rio de Janeiro.

Copyright 2014, SBGf - Sociedade Brasileira de Geofísica

Este texto foi preparado para a apresentação no VI Simpósio Brasileiro de Geofísica, Porto Alegre, 14 a 16 de outubro de 2014. Seu conteúdo foi revisado pelo Comitê Técnico do VI SimBGf, mas não necessariamente representa a opinião da SBGf ou de seus associados. É proibida a reprodução total ou parcial deste material para propósitos comerciais sem prévia autorização da SBGf.

\section{Abstract}

In this work the effect of shaliness on the physical properties of clay soils, with emphasis on the porosity of the medium was evaluated. The Nuclear Magnetic Resonance (NMR) (23 MHz Maran Ultra - Oxford Instruments, UK) was used in order to obtain absolute values of porosity in unconsolidated sediments (soil). The procedure was made with 10 synthetic samples, using different quantities of glass beads and clay in each one of then, with the intention of creating a standard grain size of the samples to be analyzed. After that we analyzed that the NMR equipment can be successfully used in measuring the petrophysical properties (porosity) of both sandy and loamy soils.

\section{Resumo}

Neste trabalho foi avaliado o efeito da argilosidade sobre as propriedades físicas de solos argilosos, com ênfase na porosidade do meio. Para isto, foi utilizado o equipamento Ressonância Magnética Nuclear (RMN) (Maran Ultra $23 \mathrm{MHz}$ - Oxford Instruments, UK), com o intuito de obter valores de porosidade absoluta em sedimentos inconsolidados (solos). No procedimento foram utilizadas 10 amostras sintéticas, usando diferentes proporções de quantidades de esferas de vidro e argila, com a intenção de criar um padrão granulométrico das amostras a serem analisadas. Podese verificar nos resultados que o equipamento de RMN pode ser utilizado com sucesso na medição das propriedades petrofísica (porosidade) de solos tanto arenosos como argilosos.

\section{Introdução}

A técnica de Ressonância Magnética Nuclear (RMN) tem sido extensivamente usada na indústria do petróleo para determinar diferentes parâmetros petrofísicos, principalmente porosidade e permeabilidade os quais são essenciais no estudo da simulação de fluxo e consequente gerenciamento de reservatórios de petróleo, gás ou água subterrânea. Aplicações importantes dessas propriedades são também encontradas em estudos de caracterização geoambiental, onde esses parâmetros têm grande impacto na avaliação de possíveis expansões de plumas contaminantes. Essas medidas são analisadas computacionalmente através de curvas de relaxações, longitudinal (T1) e transversal (T2), além do processo de inversão de dados. A RMN se destaca das demais técnicas de medidas petrofísicas, pois através dela é possível simular e compreender o comportamento natural de uma rocha junto a um fluído.

Propriedades petrofísicas de solos como porosidade, permeabilidade, superfície específica, velocidades de ondas elásticas, entre outras, são reconhecidamente afetadas pela presença de argilominerais no solo. No entanto, poucos estudos experimentais têm sido realizados no Brasil com o objetivo de quantificar esse efeito da argilosidade sobre as propriedades petrofísicas dos nossos solos.

Cupertino (2005) desenvolveu um estudo de medição das propriedades do meio poroso (porosidade $\mathrm{e}$ permeabilidade) em um conjunto de amostras de solos arenosos, e observou que os resultados experimentais eram bastante interessantes sobre o impacto dos sedimentos finos.

Diversos autores (Tosaya \& Nur, 1982; Castagna et al.,1985; Han et al.,1986; Zahaf \& Tiab, 2002) relatam o efeito da argilosidade sobre as propriedades físicas de sedimentos e de seus agregados, bem como o consequente impacto sobre as velocidades de propagação das ondas elásticas nesses materiais. Essas velocidades são fortemente dependentes da composição e da porosidade dos sedimentos. Por sua vez, a porosidade e a permeabilidade dos sedimentos são fortemente controladas pelo teor e distribuição dos argilominerais contidos nesses materiais. A quantificação ampla do efeito da argilosidade sobre sedimentos naturais é muito difícil, principalmente porque é quase impossível mostrar um mesmo tipo sedimentar com teores de argila variando de zero a cem por cento.

Por essa razão, neste trabalho foi utilizado um padrão sintético de sedimento composto por micro-esferas de vidro e com dosagens variadas em argila, afim de analisar apenas a porosidade das amostras.

\section{Metodologia}

A técnica de RMN se refere à resposta dos núcleos a um campo magnético induzido. Alguns núcleos têm um momento magnético que se comportam como se fossem barras de spins magnéticos. Estes spins podem interagir com o campo magnético induzido produzindo um sinal detectável, composto por: campo magnético estático dos imãs permanentes, campo oscilante associado com os pulsos de Rádio Frequência (RF) e flutuações do campo magnético local por elétrons desemparelhados dos núcleos vizinhos. O hidrogênio tem um momento magnético relativamente alto e é abundante tanto em água como nas moléculas dos hidrocarbonetos que se encontram em meio poroso. As quantidades medidas são: a amplitude do sinal e a taxa de relaxação. A amplitude do sinal é calibrada para dar a porosidade e os tempos de relaxação, Longitudinal (tempo de 
recuperação do campo longitudinal - T1) e Transversal, (tempo de decaimento do sinal na bobina - T2) que dão informação acerca do tamanho dos poros e das propriedades dos fluidos (Santos, 2006). Os mecanismos principais que causam relaxação longitudinal e transversal durante as aquisições de dados RMN são relaxações de superfície, volume e difusão.

Existem diversas técnicas capazes de mensurar o tempo de relaxação longitudinal (T1). A mais difundida é a denominada Inversão Recuperação (InvRec). Ela é constituída por uma sequencia de aquisições, cada uma composta por um pulso de inversão (1800) seguido por um de leitura (900), separados por período denominado tempo de recuperação da magnetização $\tau_{0}$. O parâmetro T1 é obtido através do ajuste do conjunto de pontos formado pela amplitude dos FID"s e os seus respectivos tempos de recuperação [13], de acordo com a seguinte expressão:

$$
\mathrm{I}\left(\tau_{o}\right)=\mathrm{I}_{0}\left\lfloor 1-2 e^{\left(-\tau_{0} / \mathrm{T}_{1}\right)}\right\rfloor
$$

em que $\mathrm{I}_{0}$ é amplitude do sinal de RMN com a magnetização totalmente recuperada; $\tau$ tempo de recuperação da magnetização

As medidas de relaxação spin-spin (T2) são relacionadas à presença de heterogeneidades no campo magnético $\left(\mathrm{B}_{0}\right)$, inerentes aos magnetos utilizados nos equipamentos de RMN, e faz com que os momentos magnéticos precessionem de acordo com uma distribuição de frequências, em que cada componente é denominado isocromata de spin [13]. Dessa forma, a taxa de decaimento exponencial do sinal de RMN efetivamente medida após $\mathrm{P}^{\circ} 0^{\circ} 1 / \mathrm{T}^{*}$ resulta da combinação entre as taxas de relaxação intrínseca 1/T2 da amostra e daquela gerada pela perda de coerência entre as isocromatas de spin, conforme mostra a equação:

$$
\frac{1}{\mathrm{~T}_{2}^{*}}=\frac{1}{T_{2}}+\frac{\gamma \Delta B_{0}}{2}
$$

Vale ressaltar que neste estudo foi utilizado e adquirido apenas a taxa de relaxação T2, a qual foi feita a inversão e utilizada nos resultados, conforme apresentado a seguir.

\section{Experimento e Resultados}

Para as medidas petrofísicas de porosidade absoluta das amostras sintéticas foi utilizado o equipamento RMN (Ultra $23 \mathrm{MHz}$ - Oxford Instruments, UK) o qual tem sido extensivamente usado em rochas, siliciclásticas e carbonatos, saturadas com diferentes fluídos nas áreas de hidrogeologia e Petróleo e Gás (Figura 1.1). No experimento foram utilizados 10 tubos plásticos com esferas de quartzo em tamanho de 1,4 a 1,7 mm (A150) e argila (granulometria correspondente ao passante em peneira 200 mesh) em percentuais diferenciados, sendo $0 \%$ argila e $100 \%$ de esferas, $10 \%$ argila e $90 \%$ de esferas, $20 \%$ argila e $80 \%$ de esferas, $30 \%$ argila e $70 \%$ de esferas, até chegar a $100 \%$ de argila e $0 \%$ de esferas. Para esse controle, as amostras foram pesadas controlando os percentuais dos componentes utilizados. Todas as amostras foram dopadas em água com sulfato de cobre (CuSO4) (Figura 1.2). As posições das amostras devem ser centralizadas dentro do equipamento e para isso é utilizado um calibrador em vidro (Figura 1.3). Após a calibração de centralização, a amostra é colocada dentro do equipamento para o início das medida T2 (Figura 1.4).

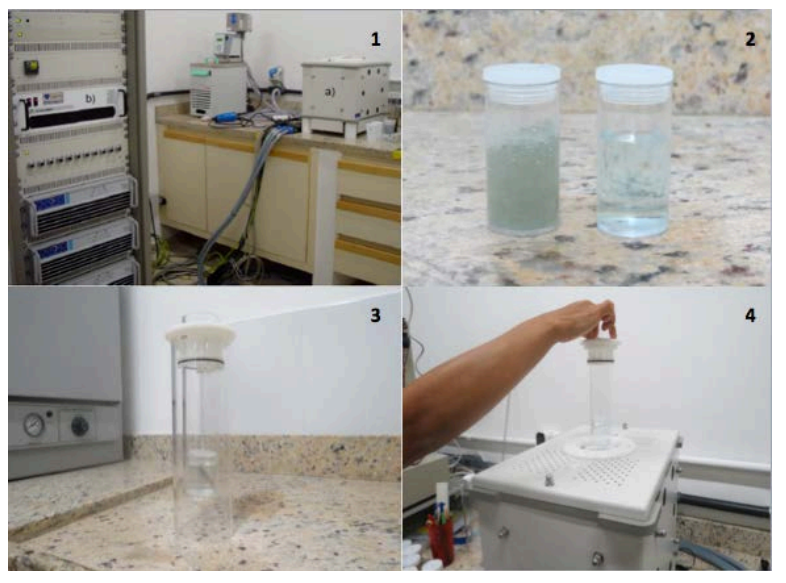

Figura 1 - 1- Equipamento Ressonância Magnética Nuclear (RMN) (Maran Ultra 23 $\mathrm{MHz}$ - Oxford Instruments, UK); 2- amostras sintéticas e água com sulfato de cobre (CuSO4); 3- tubo de ensaio com as amostras dentro de calibrador para centralização ao campo magnético ; 4- introdução do tubo de ensaio com as amostras no equipamento.

Para o início das medidas devem ser realizadas outras parametrizações de medição através dos scripts FID.exe, wobble.exe, TRN90T.exe (Figura 2) e TRN180.exe (Figura 3). Na figura 2 e demonstrado um dos gráficos obtidos para T2 e a inversão realizada. 


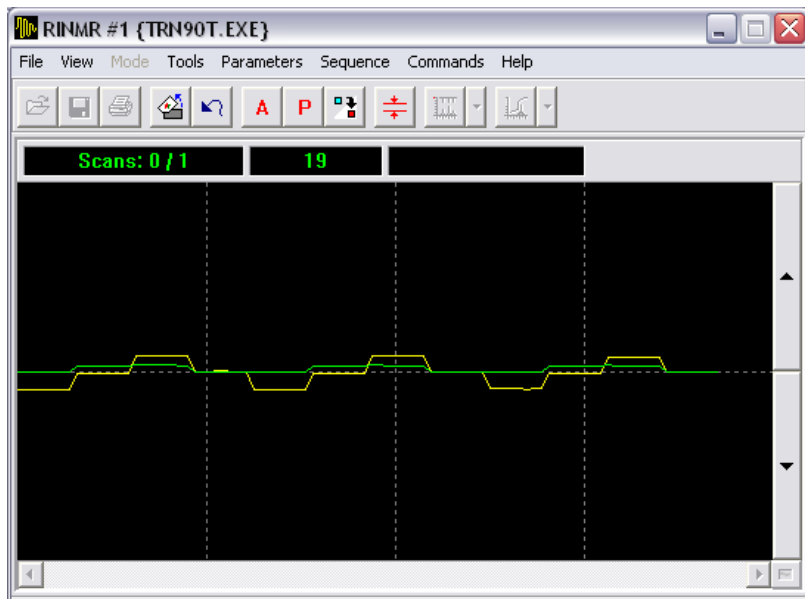

Figura 2 - Resultado da parametrização da amostragem pela TRN90T

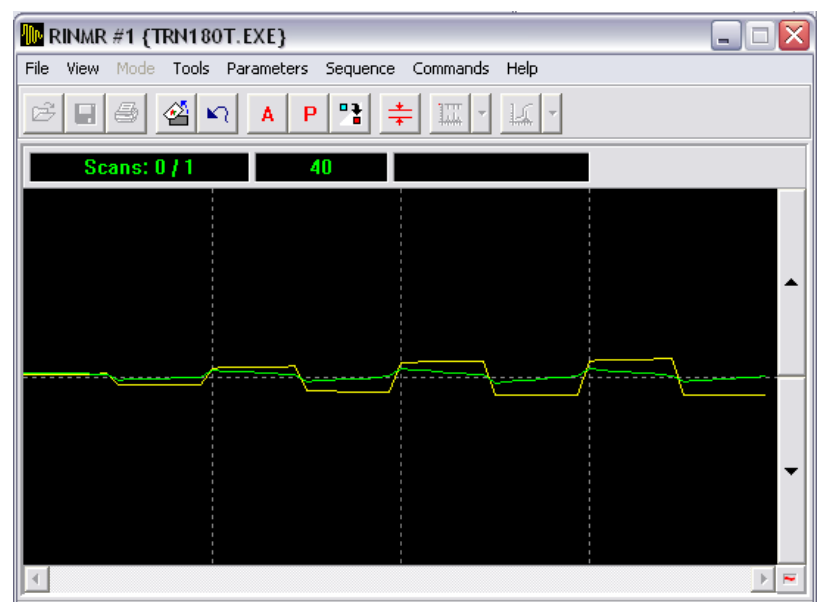

Figura 3 - Resultado da parametrização da amostragem pela TRN180T

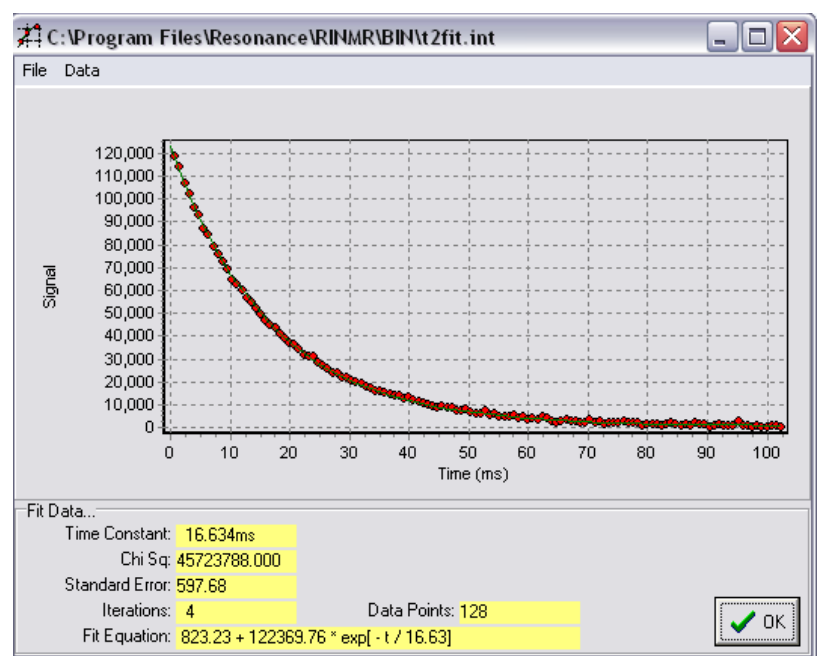

Figura 4 - Curva T2 da água dopada da amostra padrão, usada para calibração do equipamento

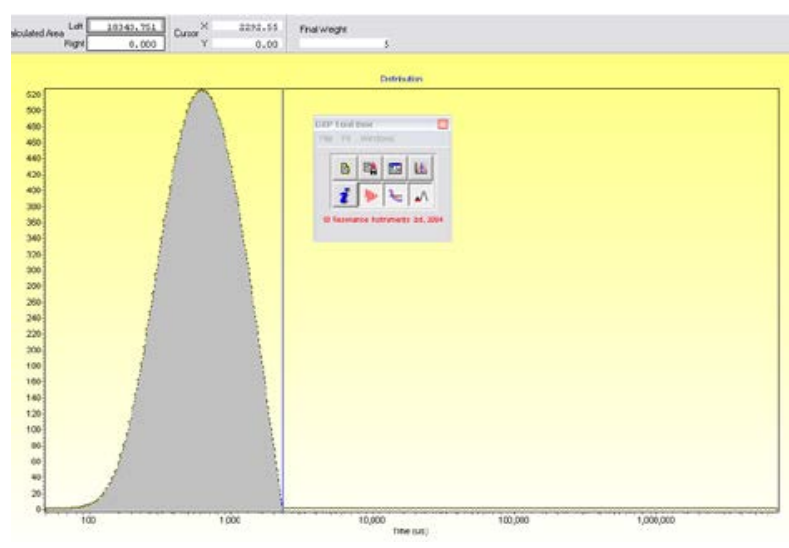

Figura 5 - Resultado da inversão dos resultados T2 apresentado após as 10 amostragens

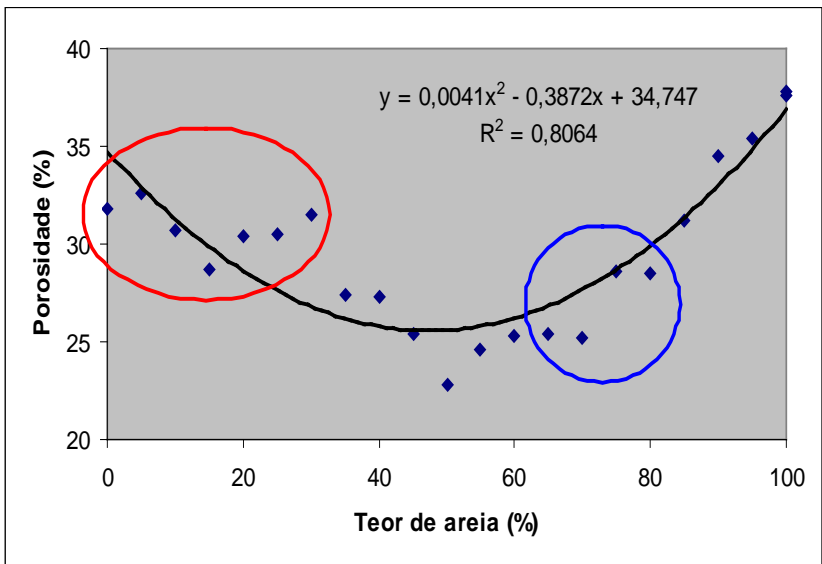

Figura 6 - Relação do teor de areia com a porosidade. A porosidade é alta em amostras com pouco ou muito teor de argila (os destaques em azul e vermelho correspondem às medidas que se afastaram a curva de ajuste quadrática, quanto mais próximo os pontos da curva melhor o ajuste $\left(R^{2}\right)$ (Carvalho et al. 2013) 


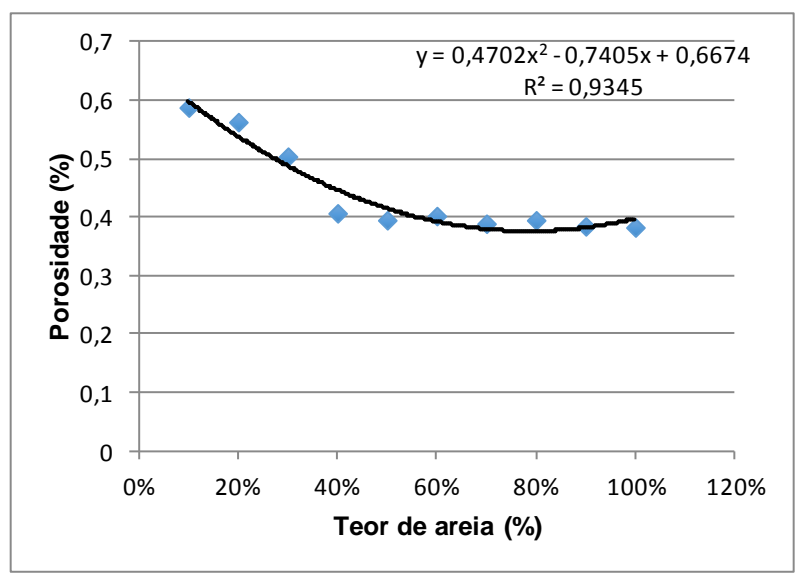

Figura 7 - Relação do teor de areia com a porosidade. A porosidade é alta em amostras com pouco ou muito teor de argila.

Conforme observado em outros estudos, notou-se que os gráficos apresentados acerca dos experimentos de porosidade seguiam um mesmo padrão, onde a linha de tendência central dos resultados apresentados é parabólica. Assim pudemos observar que no trabalho de Carvalho et al.,2013, obteve-se um gráfico oriundo de 22 amostragens de areia com água dopada no equipamento de permo-porosímetro que resultou igualmente numa linha de tendência parabólica (figura 6 ), indicando que os valores de porosidade tanto para $100 \%$ de argila quanto para $100 \%$ de areia apresentam alta porosidade e em valores de $50 \%$ argila e $50 \%$ areia apresenta baixa porosidade.

Aplicando um gráfico semelhante neste estudo obtivemos um resultado bem semelhante ao apresentado no trabalho de Carvalho et al.,2013, onde apesar deste possuir apenas 10 medições e utilizar-se do equipamento RMN (Maran Ultra $23 \mathrm{MHz}$ - Oxford Instruments, UK), pode-se ver que após a calibração do equipamento para aquisição de T2 (figura 4) e a inversão do mesmo (figura 5), este igualmente atingiu o resultado esperado formando um linha de tendência parabólica (figura 7), apresentando o resultado confirmado anteriormente.

Dessa forma podemos perceber que a técnica de RMN, a qual foi implementada neste trabalho possuí uma importante serventia, provendo bons resultados, podendo ser extensamente implementada em outros estudos de porosidade, tal qual o permo-porosímetro, afim de descobrir como ocorre essa interação de rochas sedimentares com o fluxo de fluídos.

\section{Discussão e Conclusões}

A utilização do equipamento Ressonância Magnética Nuclear (RMN) (Maran Ultra $23 \mathrm{MHz}$ - Oxford Instruments, UK) pode ser aplicado para a medição de propriedades físicas de solos argilosos e arenosos, isso porque este alcançou bons resultados. Dessa forma o experimento mostrou que a porosidade é elevada nas amostras sintéticas, sejam elas com grande ou pouca quantidade de argila. Após medirmos os sinais captados e a taxa de relaxação T2 e aplicarmos a inversão no resultado das 10 amostras tornou-se evidente que:

- A porosidade se mantém alta quando apresenta-se altos teores de argila e areia nas amostras.

- A porosidade decai exponencialmente quando os teores de argila e areia caem pra $50 \%$ ou menos.

- A densidade diminui linearmente com a argilosidade, enquanto que a porosidade decresce exponencialmente com a diminuição da densidade.

Com isso, pode-se então mostrar que a comparação dos teores de argila e areia com a porosidade resultante mostrou-se diretamente proporcional, isso devido à granulometria e a seleção dos grãos. Assim, foi através desse estudo que pode-se verificar as relações das propriedades físicas em amostras sintéticas de solos, com variações percentuais de argila, já que possuímos diferentes tipos de solos com diferentes variações granulométricas.

\section{Agradecimentos}

Ao Laboratório de Geofísica e Petrofísica da UFRJ.

\section{Referências}

Alvarez J.P. G. 2007. Effect of Microstructure and Pore Fluid on the Elastic Properties of Carbonate Rocks. A Thesis Submitted To The Graduate Faculty Master Of Science Norman, Oklahoma.

Dastidar R. 2004. Integrating NMR with other petrophysical parameters to characterize a turbidite reservoir. Thesis approved for the Mewbourne school of petroleum and geological engineering.

Talabi O. A. 2008. September. Pore-Scale Simulation of NMR Response in Porous Media. A dissertation submitted to the Department of Earth Science and Engineering of Imperial College Lodon In Partial Ulfilment 
of The Requirements for the Degree Of Doctor Of Philosophy.

Toumelin E. 2002. Monte Carlo Simulation of Nuclear Magnetic Resonance Measurements in Carbonate Rocks Under a Constant Magnetic Field Gradient. A dissertation submitted the University of Texas at Austin.

Rabelo S. K. L. 2004. Estudo Sobre a Viabilidade do Cálculo da Porosidade com Base em um Conjunto Reduzido de Perfis e Sua Aplicação na Bacia de Almada/Ba. Dissertação de M.Sc, Universidade Estadual do Norte Fluminense - UENF, Campos, RJ, Brasil.

Carvalho M. R. T, Dias G. A. N, Soares J. A. 2013. Estudo experimental do efeito da argilosidade sobre as propriedades físicas de solos.

Cupertino D.F. 2005. Caracterização de propriedades do meio poroso em solo arenoso através de pemoporosímetro a gás, Graduação em Geologia, Universidade Federal do Rio de Janeiro, monografia, 61 p.

Dvorkin et al. 2011. Relevance of Computational Rock Physics. Gophysics. Vol 76. N 55 (September-October 2011) E141-E153, 31 Figs. 